\title{
A model of defect cluster creation in fragmented cascades in metals based on morphological analysis
}

\section{De Backer, A.}

2018-10-10

De Backer , A, Domain , C , Becquart , C S , Luneville , L, Simeone , D , Sand , A E \& Nordlund, K 2018 , ' A model of defect cluster creation in fragmented cascades in metals based on morphological analysis ' , Journal of Physics. Condensed Matter , vol. 30 , no. 40 , 405701 . https://doi.org/10.1088/1361-648X/aadb4e

http://hdl.handle.net/10138/307857

https://doi.org/10.1088/1361-648X/aadb4e

cc_by_nc_nd

acceptedVersion

Downloaded from Helda, University of Helsinki institutional repository.

This is an electronic reprint of the original article.

This reprint may differ from the original in pagination and typographic detail.

Please cite the original version. 


\title{
Morphological transition of collision cascades in high energy ion and neutron impacts
}

\author{
A. De Backer ${ }^{1}$, C. Domain ${ }^{2}$, C.S. Becquart ${ }^{3}$, L. Luneville ${ }^{4,5}$, D. Simeone ${ }^{5,6}$, A. E. Sand ${ }^{7}$, and
} K. Nordlund ${ }^{7}$

${ }^{1} \mathrm{CCFE}$ - Culham Centre for fusion Energy, Abingdon, Oxon OX14 3DB, United Kingdom ${ }^{2}$ EDF Lab Les Renardières, Dpt MMC, F-77250 Moret sur Loing, France

${ }^{3}$ Univ.Lille, CNRS, INRA, ENSCL, UMR 8207, UMET, Unité Matériaux et Transformations, F 59000 Lille, France

${ }^{4}$ CEA/DANS/DMN/SERMA/LLPR-LRC CARMEN, CEA Saclay 91191 Gif sur Yvette, France

${ }^{5}$ Centralesupelec/SPMS/LRC CARMEN, 92292 Chatenay Malabry, France

${ }^{6}$ CEA/DANS/DMN/SRMA/LA2M-LRC CARMEN, CEA Saclay 91191 Gif sur Yvette, France

${ }^{7}$ Department of Physics, University of Helsinki - P.O. Box 43, FI-00014 Helsinki, Finland

February 16, 2018

\begin{abstract}
The impact of ions and neutrons in materials causes cascades of atomic collisions that expand and shrink, leaving microstructure defect debris, i.e. interstitial or vacancy clusters or loops of different sizes. In [A. De Backer, A. E. Sand, K. Nordlund, L. Lunéville, D. Simeone, and S. L. Dudarev. EPL, 115(2):26001, 2016.], we described a method to detect the cascade fragmentation in subcascades and a model of primary damage combining the Binary Collision Approximation (BCA) and Molecular dynamics (MD). In this paper including $\mathrm{W}, \mathrm{Fe}, \mathrm{Be}, \mathrm{Zr}$ and 20 other metals, we demonstrate that the fragmentation energy increases with the atomic number and decreases with the atomic density following a unique power law. Above the fragmentation energy, the cascade morphology exhibits a second evolution and consists in branches decorated by chaplets of small subcascades that we characterize with the cross pair correlation functions of the multipoint patterns formed by the subcascades. We then derive the number of subcascades that interact in high energy cascades. The subcascade interaction is introduced in our model of primary damage by adding pairwise terms. Using statistics obtained on hundreds of MD cascades in Fe from, we demonstrate that the interaction of subcascades increases the fraction of damage in clusters created by high energy cascades, which can impact the long time evolution under irradiation. The enhanced formation of large self-interstitial (SIA) defects due to subcascades interactions is consistent with the shock-wave interactions reported to cause such effect in [A.F. Calder, D.J. Bacon, A.V. Barashev, and Yu.N. Osetsky, Philosophical Magazine, 90(7-8):863884, 2010.]. Finally, we apply our model to predict the cluster size distribution of $500 \mathrm{keV}$ Fe cascades where large MD statistics is not feasible.
\end{abstract}

\section{Introduction}

In fission and fusion nuclear installations, structural materials are exposed to different and non-homogeneous ion and or neutron fluxes. One challenging task is the characterization of the primary damage, i.e. the defects of the microstructure created by the displacement cascades of energy varying from a few tens of eV to $\mathrm{MeV}$ in metals including Fe, Zr, W, Be and alloys. It is established that the number, the type and the size distribution of the defects created by cascades depend on the material and the particle energy [1]. The prediction of the formation of large defects is a crucial aspect of the source term of the long term evolution models [2]. In the frame of the multiscale modelling, numerous approaches are pursued such as Molecular Dynamics (MD) and 
the Binary Collision Approximation (BCA). The rationalization of their results is essential to the large scale models taking into account the spatial in-homogeneity of the installation and the neutron spectrum [3, 4].

The works reported in $[5,6,7,8,9,10]$ showed that an energy exists above which cascades form subcascades and that the fragmentation energy depends on the materials. These studies covered a large range of atomic numbers, atomic densities, several crystal structures and displacement threshold energies. Furthermore, they raised the question of the overlap or the vicinity of subcascades and their possible interaction. Interestingly, the formation of large clusters at interfaces between subcascades has been observed in MD cascades in Fe and explained by shock-wave interaction in $[11,12]$.

In $[13,10]$, we showed the fractal nature of cascades in the frame of the BCA and demonstrated that it naturally introduces power laws in the total number of defects. In [14], we revealed the existence of a power law in the frequency of defect clusters as a function of their size. Recently [15], we proposed a new method of decomposition of cascades in subcascades and a model of defect cluster production of Primary Knocked-on Atoms (PKA) of any energy. The frequency of the defect clusters in individual subcascades is multiplied by the frequency of these subcascades in the large fragmented cascade. The interest of our approach is that the subcascade frequency can be efficiently calculated using the BCA and that full MD cascade calculations are only necessary up to the fragmentation energy. We also demonstrated in [16] that this explains the deviation from the power law of the number of loops per ion as a function of the loop size, observed in experiments of $150 \mathrm{keV}$ self irradiation of $\mathrm{W}$ at cryogenic temperature [17].

In this work, we extend our cascade decomposition study to other metals and to MD cascades. Afterwards, cascades are seen as random multitype point patterns formed by the subcascades, the type of which is given by their volume. Using the cross pair correlation functions, we derive the number of interacting subcascades and we include it in our model of primary damage. Our model is adjusted on MD cascades and extrapolated to high energy cascades that are hardly accessible to MD.

In the first section we describe the computational methods for the BCA and MD cascades.

The second section contains the comparison of the subcascade decomposition of BCA and MD cascades and the discussion on the choice of the cell size and the energy criterion $E_{c}$.

In the third section, using BCA cascades in various metals, we demonstrate that with the strictest criterion $E_{c}=0$, the fragmentation energy follows a simple power law of the atomic number and the atomic density. We propose a choice of $E_{c}>0$ based on simple thermodynamic properties of metals and show that it reduces the fragmentation energy of high melting point metals, particularly high $\mathrm{Z}$ ones like $\mathrm{W}$ where cascades are very compact.

In the fourth section we describe the morphological evolution of cascades taken as multitype point patterns where each subcascade is one point, the type of which is given by the subcascade volume. The cascade morphology is captured using the cross pair correlation functions where pairs are formed by subcascades of different volumes. New aspects of the transition above the fragmentation energy are described in terms of number of pairs of nearby subcascades. The subcascades at close distance from each other are the ones susceptible to interact. We determine the evolution of the number of interacting pairs of subcascades as a function of the PKA energy, revealing features of the cascade branching.

In the last section, we develop our model of primary damage of high energy cascades. Our model sums the defect production in individual subcascades and the pairwise terms of the subcascade interactions. The model is adjusted on full MD cascades in Fe from $1 \mathrm{keV}$ to $80 \mathrm{keV}$. We demonstrate that our model can predict the creation of large SIA clusters due to the subcascade interaction. We finally apply our model to the prediction of the primary damage of high energy cascades which is not feasible by full MD so far, for example, the 500 keV Fe self implantation performed in our TriCEM project.

\section{Computational method of BCA and MD cascades}

BCA cascades are calculated using SDTrimSP [18], the slowing down of the projectile is modelled as described in [19] with no consideration of crystal structure by a series of random collisions and a continuous interaction with electrons. The main material parameters are the atomic number and atomic density. The slowing down of the knock-on atoms and the development of the cascade is obtained similarly with no interaction between moving atoms. No free surface is included in this work, and we will use the terminology PKA to refer to the initial projectile. The ZBL potential $[20,21]$ is used along with the electronic stopping power description of Oen and Robinson [22]. In [23, 24], the effect of the model of electronic stopping power changes the defect creation in high energy cascades. But this is out of the scope of the BCA approach and this paper. Similarly, we found that the effect of the threshold displacement energy and other parameters of the BCA model are not significant here. The main reason is that in our decomposition method, the features of the cascades are averaged in cubic cells of $1 \mathrm{~nm}$ size causing a coarse-graining which reduces the effect of some parameters of 
the BCA. In other words, changing the BCA model parameters, except the atomic number or atomic density, will modify the cascade features only at size scales, too small to impact the results of the decomposition method we propose here. Notice that the BCA models, do not treat the melting and recrystallisation as MD does. BCA models alone cannot account for the defect debris remaining after the cascade cooling down, the recovery of the lattice and the recombination of vacancy and interstitial defects.

For this work, we used MD cascades in Fe described in [25]. They have been performed using DYMOKA [26] with the Embedded Atom Model potential of Ackland et al. [27] hardened with ZBL [20]. We characterized the validity of this potential to model atomic collisions in the range of the threshold displacement energy by comparison with ab initio MD in [28]. Here, classical MD simulations have been done within the NVE ensemble with periodic boundary conditions with up to 3.456 .000 atoms (box of $120 \times 120 \times 120$ cubic cells). After a thermalisation stage of 3 ps at $100 \mathrm{~K}$, the kinetic energy is given to a PKA. The time step is adjusted during the simulation which lasts 20 ps and no electronic loss is considered. Large statistics have been obtained with several hundreds of cascades per energy up to $120 \mathrm{keV}$ (800 to 1000 cascades in most of the cases). Half of the cascades were initiated with a $\langle 135\rangle$ PKA direction and half with a random direction (no statistical differences were observed). To detect vacancies and SIA defects at the end of the simulation, lattice site analysis is performed. A cluster is defined by all entities (vacancy and SIA) within a critical distance (lattice parameter, e.g. second nearest neighbour distance), and the net defect sum determines the cluster size which is used for the statistics of defect size.

\section{Cascade fragmentation}

In our decomposition method, the energy losses at the end of the BCA cascade are projected and averaged on a cubic tessellation of space. The energy density exhibits fluctuations with hot cores separated by low energy interfaces. A criterion of minimum energy density, $E_{c}$, distinguishes what is part of the cascade or not and reveals the interfaces between subcascades. The level of details of the cascade is adapted adjusting the cubic cell of the space tessellation and $E_{c}$. The subcascades are formed by coalescence of neighbouring cells. The presence of an interface between subcascades means that a contiguous volume exists with a thickness of at least one cell. As opposed to other methods based on the tracking of high energy knock-on atoms, our is an effective decomposition method, no subcascade overlap is possible, but the subcascade vicinity can be investigated. Figure 1 shows a picture of one $100 \mathrm{keV}$ Fe cascade analysed with different cell sizes and the average number of subcascades as function of the cell size for different PKA energies (using $E_{c}=44$ $\mathrm{eV} / \mathrm{nm}^{3}$ ). A simple visualization suggests an optimum of the cell size around $1 \mathrm{~nm}$. Small cells keep too much description of the cascade and projected on large cells, the cascade energy is too diluted. The plot of Figure $1 \mathrm{~d}$ reveals that the number of subcascades exhibits a plateau for cell size of 1 to $2.5 \mathrm{~nm}$ for the whole energy range studied here. This shows that $1 \mathrm{~nm}$ is a good choice to capture the cascade morphology. We will use this value for the results of the next sections. 


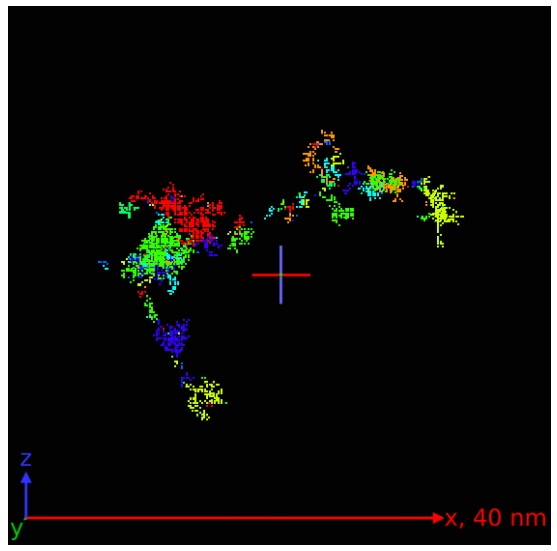

(a)

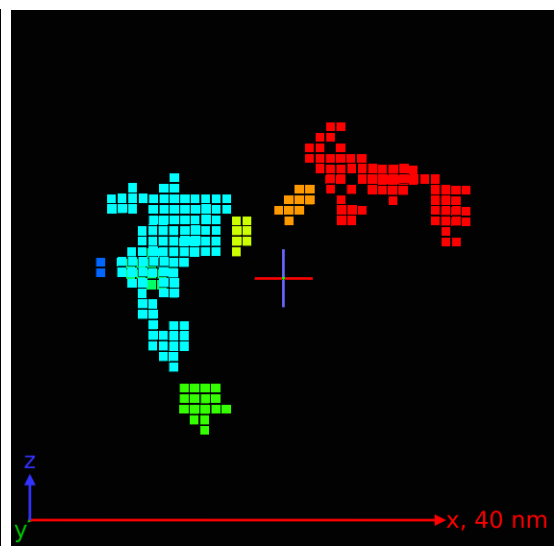

(b)

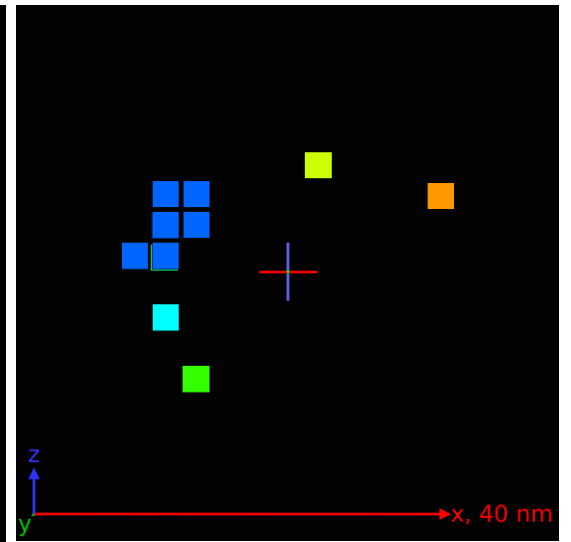

(c)

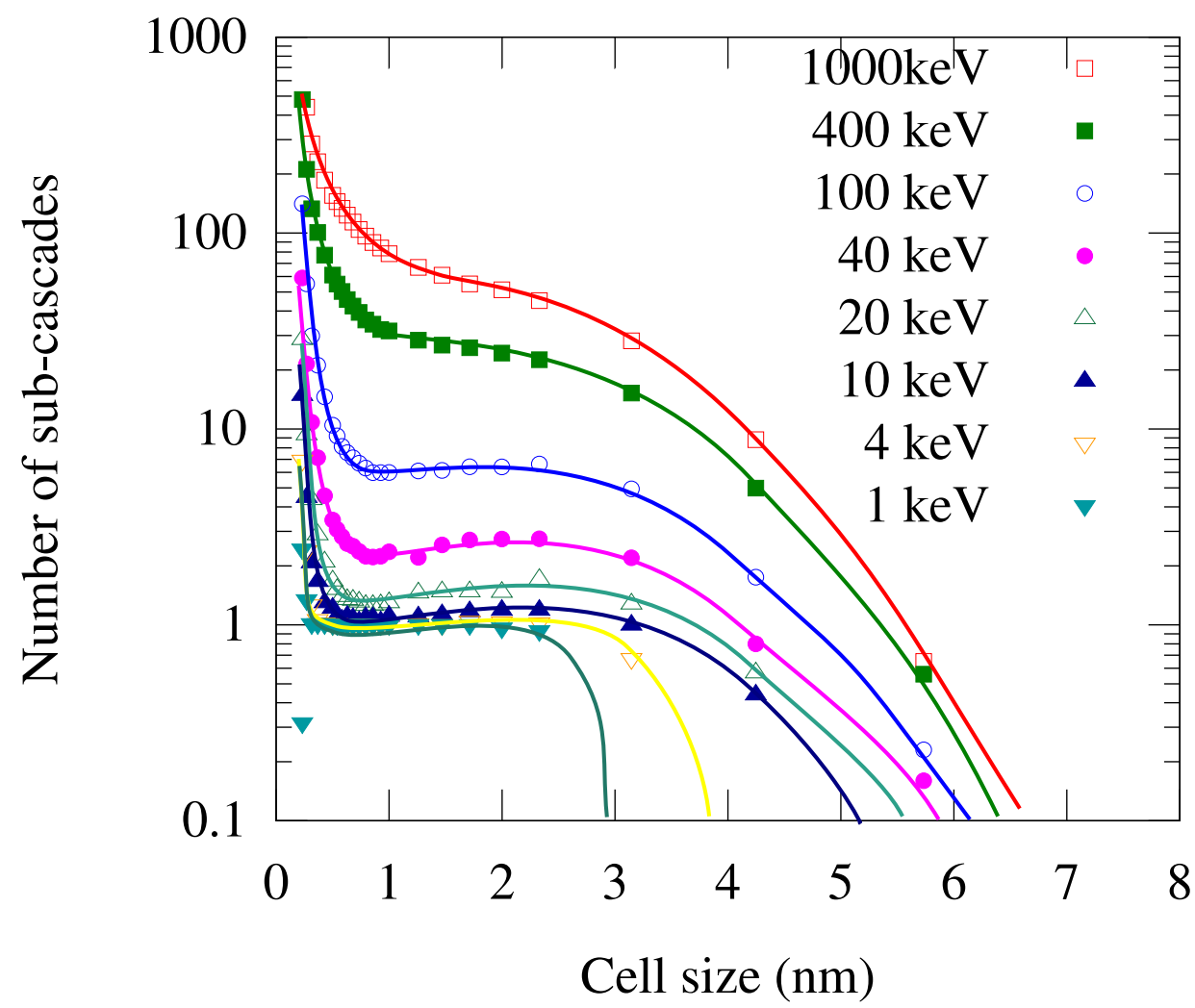

(d)

Figure 1: (a-c) Pictures of one $100 \mathrm{keV}$ BCA cascade in Fe analysed with increasing cell sizes, $0.2 \mathrm{~nm}, 1 \mathrm{~nm}$ and $3 \mathrm{~nm}$. (d) Averaged number of subcascades as a function of the cell size and the PKA energy in Fe.

We applied the same space tessellation and energy procedure to MD cascades, recording the kinetic energy of the atoms with kinetic energy larger that $0.4 \mathrm{eV}$. With MD cascades, the subcascade decomposition depends on time. After the ballistic stage, the cascade cools down and no more hot region is detected. The average volumes of $20 \mathrm{keV} F$ cascades as a function of time using BCA and MD with various cell size and $E_{c}$ are plotted in Figure 2a. During the ballistic phase that lasts around $0.1 \mathrm{ps}$, the volume of BCA and MD cascades increases linearly with a rate that is mainly affected by the cell size for the BCA and $E_{c}$ for MD. The smaller the cell size, the larger the volume. This is explained by the fractal feature of the BCA cascades. On the other hand, in MD cascades atomic low energy interactions make our method more sensitive to $E_{c}$ in the layer around the subcascade hot cores (see sketch drawn in Figure 2b). We must find a compromise. With large $E_{c}$, only the hottest cores are detected and the total volume is small. On the other hand with small $E_{c}$, subcascades are not distinguished. More description of the thermal processes in MD is out the scope of this paper. It remains that after the ballistic stage, the BCA cascades do not evolve any more when the MD cascade volume decreases because of the cooling down. The decomposition method is applied when the volume is maximum, the time of which depends mainly on the PKA energy. 


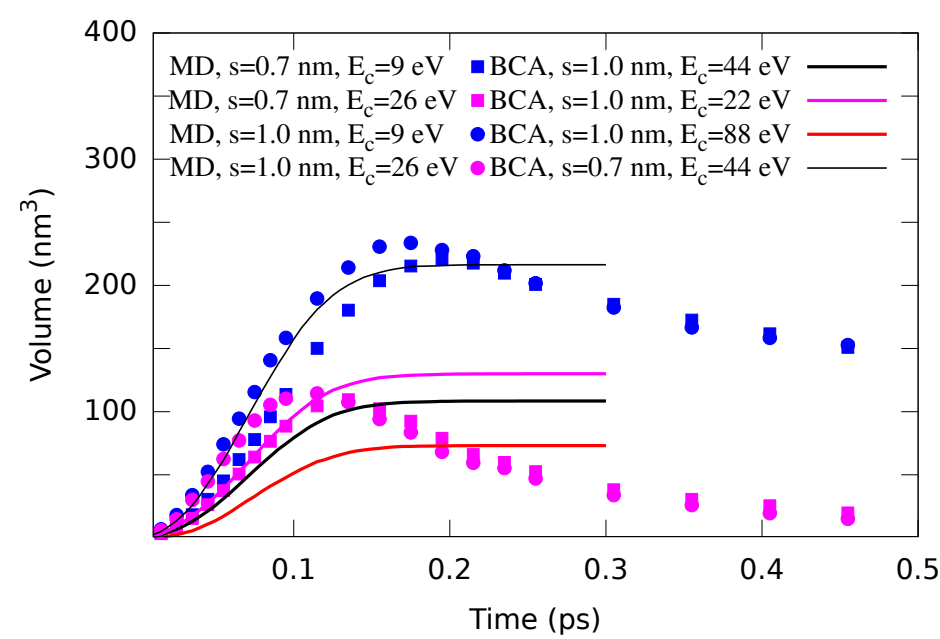

(a)

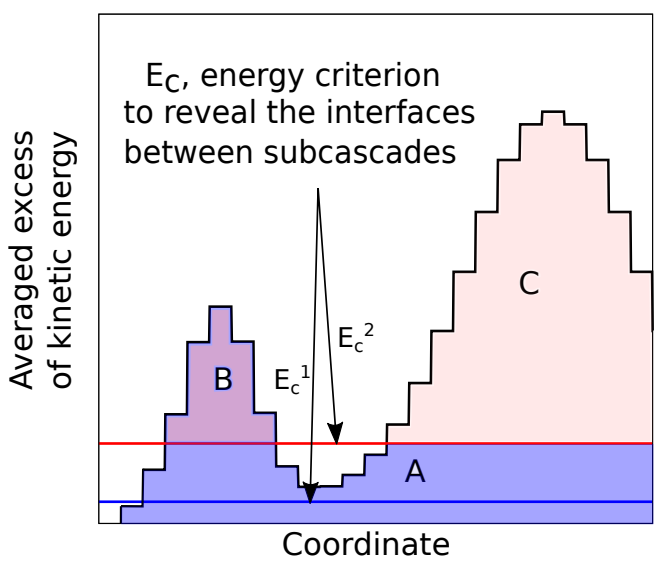

(b)

Figure 2: (a) Cascade volume as function of time for $20 \mathrm{keV}$ cascades calculated by BCA and MD and similarly analysed with various cell sizes, $s$ and $E_{c}$. (b) Schematic drawing of the effect of $E_{c}$ which reveals the interface between subcascades: with the small $E_{c}^{1}$, only one subcascade $\mathrm{A}$ is detected and with the larger value $E_{c}^{2}$, two subcascades B and C are detected but with too large value, small subcascades are missed.

The evolution of the number of subcascades as function of the PKA energy has been calculated and averaged on 1500 BCA cascades per energy and several hundreds MD cascades in Fe. The number of subcascades, $n_{S C}(E)$, starts as one, with one main volume, then becomes progressively a linear function of the PKA energy which can be approximated by

$$
n_{S C}(E)=\left\{\begin{array}{cc}
1 & \text { if } E \leq E_{f r} \\
E / E_{f r} & \text { if } E>E_{f r}
\end{array}\right.
$$

which defines $E_{f r}$, the fragmentation energy. The fragmentation energy depends slightly on the cell size and $E_{c}$ (see in Figure 3). Both MD and BCA predict that fragmentation starts between 10 and $15 \mathrm{keV}$. Notice that without the high level of statistics of subcascade numbers, for example from a limited number of MD simulations, the point where the number of subcascades equals 2 could be seen as the threshold for subcascade splitting, and our results are in agreement with Stoller's paper [29], which gives the threshold for splitting in Fe as 20-30 keV. The progressive increase of the number of subcascades is due to the occurrence of small subcascades that decorate the main volume. In the linear part, no characteristic volume exists and the frequency of subcascades as function of their volume is a power law in favour of the small volume. Notice that the subcascade frequency vanishes for a volume close to the volume corresponding to the fragmentation energy (a thorough description is given in [15]). We determined that in Fe, $E_{f r}=15 \mathrm{keV}$ and $V_{f r}=90 \mathrm{~nm}^{3}$. 


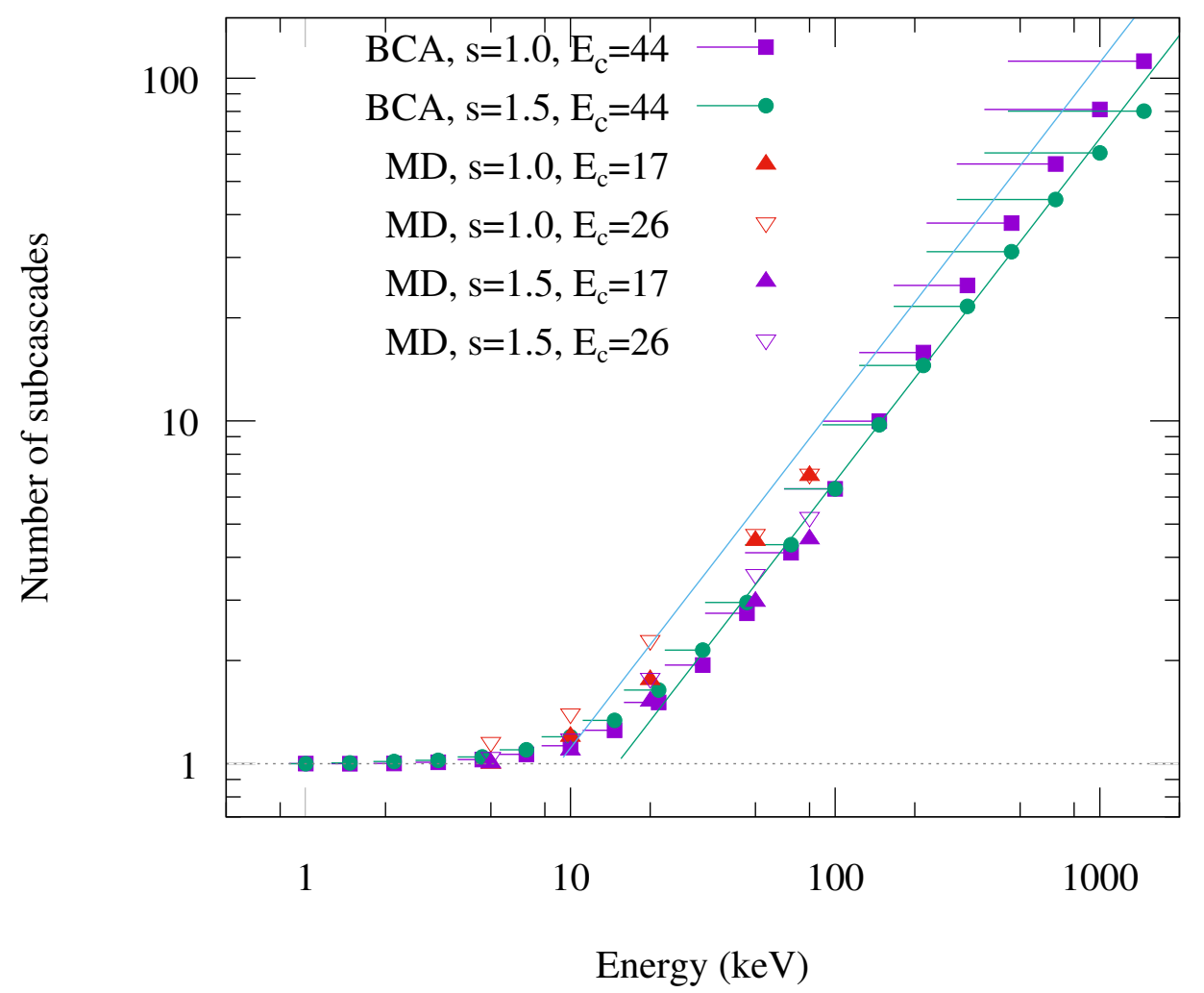

Figure 3: Number of subcascades detected by $\mathrm{MD}$ and BCA as a function of the PKA energy for various combination of the cell size $s$ (in $\mathrm{nm}$ ) and the energy criterion $E_{c}$ (in $\mathrm{eV} / \mathrm{nm}^{3}$ ), in Fe. The fragmentation energy defined by equation (1) is between $10 \mathrm{keV}$ and $15 \mathrm{keV}$. Notice that the electronic losses have been included in the BCA model and not in the MD one. Lines indicate the energy shift to subtract the electronic losses, which enhances the agreement between MD and BCA.

\section{Fragmentation energy within the periodic table}

In this section, we will use both $E_{c}=0$ and a simple expression (that we already proposed in [15]) that depends on thermodynamic properties of the material,

$$
E_{c}=\left(C\left(T_{f}-T\right)+L\right)
$$

where $C$ and $L$ are the specific heat and the enthalpy of fusion respectively, $T_{f}$ and $T$ are the melting point and the material temperature. The justification is that the melting of the crystal is observed in the hot cores of the cascades and that the formula (2) is a convenient estimate of the energy required to melt the material. The exact amount of energy required to melt a cubic cell of material is a more complex question. In particular, displacement cascades are not equilibrium processes. The mechanical (superheating) melting temperature is about $15 \%$ to $20 \%$ larger than the melting temperature and the crystal melts immediately with no enthalpy of fusion [30,31,32,33]. Furthermore as the specific heat does evolve with temperature, an integral form is more precise. Following [34], one sees that the proper integration from room temperature to melting temperature leads to a 2 times lower energy compared to our expression. These aspects are out of the scope of this paper, however, we wish a formula easily applicable to many metals. Values of $E_{c}$ for Be, $\mathrm{Fe}, \mathrm{Zr}, \mathrm{Mo}$ and $\mathrm{W}$ are given in table $1 . \mathrm{W}$ and Mo require the largest values of $77 \mathrm{eV}$ and $66 \mathrm{eV}$ because of their high melting point and $\mathrm{Zr}$ has the lowest value because of its low atomic density. Be has a low melting point but this is compensated by its high atomic density.

BCA Cascades were done in 22 metals with PKA energy ranging from $1 \mathrm{keV}$ to $1 \mathrm{MeV}$. 200 cascades per energy was a sufficient statistics. All metals present a transition from one single domain to an increasing number of subcascades. The fragmentation energy is obtained by adjustment of equation (1). In our study, the main parameters differing one material from another are the atomic number, $Z$, the atomic density, $d$ and thermodynamic properties in the energy criterion, $E_{c}$. 


\begin{tabular}{cccc|cc} 
Atomic number & $\begin{array}{c}\text { Atomic density } \\
\left.\text { [atom per } \mathrm{nm}^{-3}\right]\end{array}$ & $\begin{array}{c}\mathrm{E}_{c} \\
{\left[\mathrm{eV} / \mathrm{nm}^{3}\right]}\end{array}$ & $\begin{array}{c}\mathrm{E}_{f r}^{\max } \\
\mathrm{keV}\end{array}$ & $\begin{array}{c}\mathrm{E}_{f r} \\
\mathrm{keV}\end{array}$ \\
\hline $\mathrm{Be}$ & 4 & 123 & 37 & 0.75 & 1.1 \\
$\mathrm{Al}$ & 13 & 60 & 11 & 4 & 1.8 \\
$\mathrm{Fe}$ & 26 & 85 & 44 & 45 & 15 \\
$\mathrm{Ni}$ & 28 & 91 & 51 & 58 & 21 \\
$\mathrm{Zr}$ & 40 & 43 & 30 & 20 & 9 \\
$\mathrm{Mo}$ & 42 & 65 & 66 & 84 & 17 \\
$\mathrm{~W}$ & 74 & 63 & 77 & 315 & $75^{*}$
\end{tabular}

Table 1: Atomic number, atomic density, energy criterion for $1 \mathrm{~nm}$ cells $\left(E_{c}\right)$, maximum fragmentation energy $\left(E_{f r}^{\max }\right.$, with $\left.E_{c}=0\right)$ and the fragmentation energy with $E_{c}>0$ given by equation (2). ${ }^{*}$ This value is smaller than in [15] because of the choice of equation 1.

We first described the effect of the atomic number and the atomic density by repeating the BCA cascade decomposition taking $E_{c}=0$. In this case, the criterion is the strictest and the fragmentation energies are maximum. The atomic number varies from 4 for the Be, to 92 for $\mathrm{U}$ and increases the fragmentation energy. The atomic density varies from 43 at. per $\mathrm{nm}^{3}$ for $\mathrm{Zr}$ to 123 at. per $\mathrm{nm}^{3}$ for Be and also increases the fragmentation energy. Interestingly, the density of metals (after Be which has only 2s electrons per atom) evolves as three successive bell shape curves with the filling of the successive electronic bands $3 \mathrm{~d}, 4 \mathrm{~d}$ and $5 \mathrm{~d}$ as explained in [35]. The bell shapes is actually visible on the fragmentation energy when one sweeps along the periods of the periodic table because of the effect of the atomic density.

In our previous work [36], we observed that the fragmentation energy follows a power law as function of the atomic number when the density was constant. Using our new approach and including the effect of the atomic density, we found that the fragmentation energy still follows a power law,

$$
E_{f r}^{\max }=E_{1}\left(Z \frac{d}{d_{F e}}\right)^{q_{c}}
$$

but depending on an effective atomic number defined by $Z d / d_{F e}$, where $Z$ is the atomic number and $d$ is the atomic density (with $\mathrm{Fe}$ as reference material). Our results and this expression using $E_{1}=0.013 \mathrm{keV}$ and $q_{c}=2.5$ are plotted in Figure 4a. Notice that the maximum fragmentation energy is large, $45 \mathrm{keV}$ for $\mathrm{Fe}$ and $315 \mathrm{keV}$ for $\mathrm{W}$ for example. The reason is that $E_{c}=0$ is a too strict criterion, it requires an interfaces of 1 $\mathrm{nm}$ thick with no collision between two subcascades.

The fragmentation energy with $E_{c}>0$ is plotted in Figure $4 \mathrm{~b}$. It varies between $2 \mathrm{keV}$ and $500 \mathrm{keV}$ depending on the metal and follows a sawtooth evolution as a function of the atomic number of metal groups $2 \mathrm{~s}, 3 \mathrm{p}, 3 \mathrm{~d}, 4 \mathrm{~d}, 5 \mathrm{~d}$ and $5 \mathrm{f}$ because of the combined effect of the atomic density and $E_{c}$. The decrease of the density is compensated by a low $E_{c}$ (due to low melting point) for most metals of the end of each period, columns 11 and 12 of the periodic table, Cu, Zn, Ag, Cd, Pt and Au. The fragmentation energy is $15 \mathrm{keV}$ for Fe and $75 \mathrm{keV}$ for $\mathrm{W}$, which is in agreement with full MD cascades and experimental results [16]. Notice that this last value is divided by 2 compared to our previous, work where we defined the fragmentation energy by the energy where cascades form two subcascades.

Figure $4 \mathrm{~b}$ represents the fragmentation energies using the fractal approach $[13,36]$ and other methods from $[7,8,9]$. The general tendency of the effect of the atomic number is visible. However, in our work and proposing the equation 3, we separated the effect of the atomic density and the atomic number. The main difference between the method of [7] and our is our energy criterion which reduces (resp. increases) the number of subcascades for metals with a a large $E_{c}$, i.e. a high (resp. low) melting temperature or heat capacity for example. Notice that our method is an effective method where no cascade overlap is possible, contrary to Ref. [8, 9]. Interestingly Heinish's method using MARLOWE [37] takes into account the crystal structure unlike SDTrimSP. The authors noticed that the reduced fragmentation energies tend to group by crystal structure but the effect of the atomic density was not rationalized. These methods also sometimes differ by the choice of the interaction potential, ZBL and Moliere potentials, the later produces a larger collision cross section at low energy, i.e. should predict more dense cascades and consequently larger fragmentation energy.

Our study of BCA cascades proves that the fragmentation energy is first a function of the atomic number, then the atomic density, and also depends on the thermodynamic properties. Further works are necessary to fully confirm the effect of the thermodynamic properties and determine the effect of the interatomic potentials. 


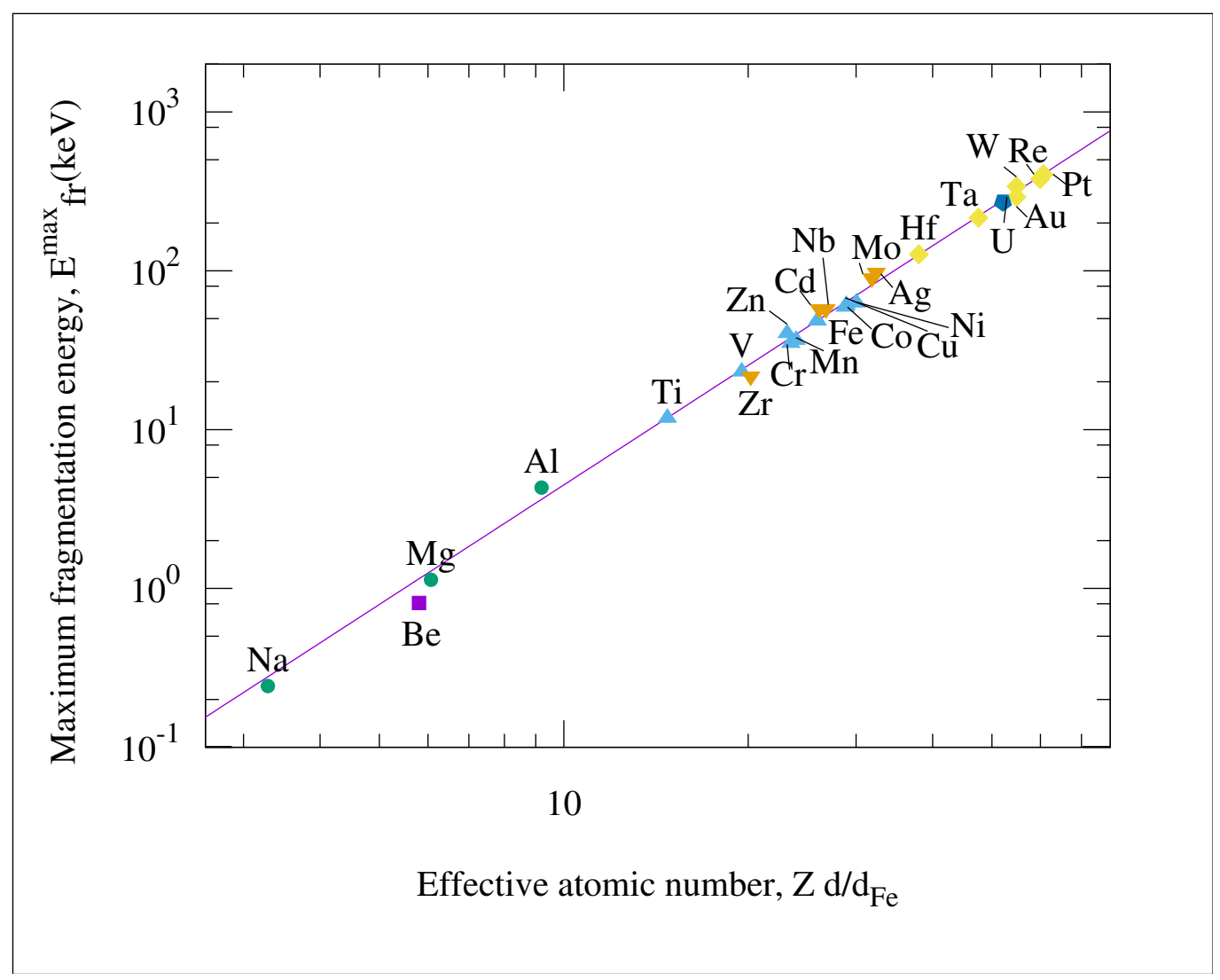

(a)

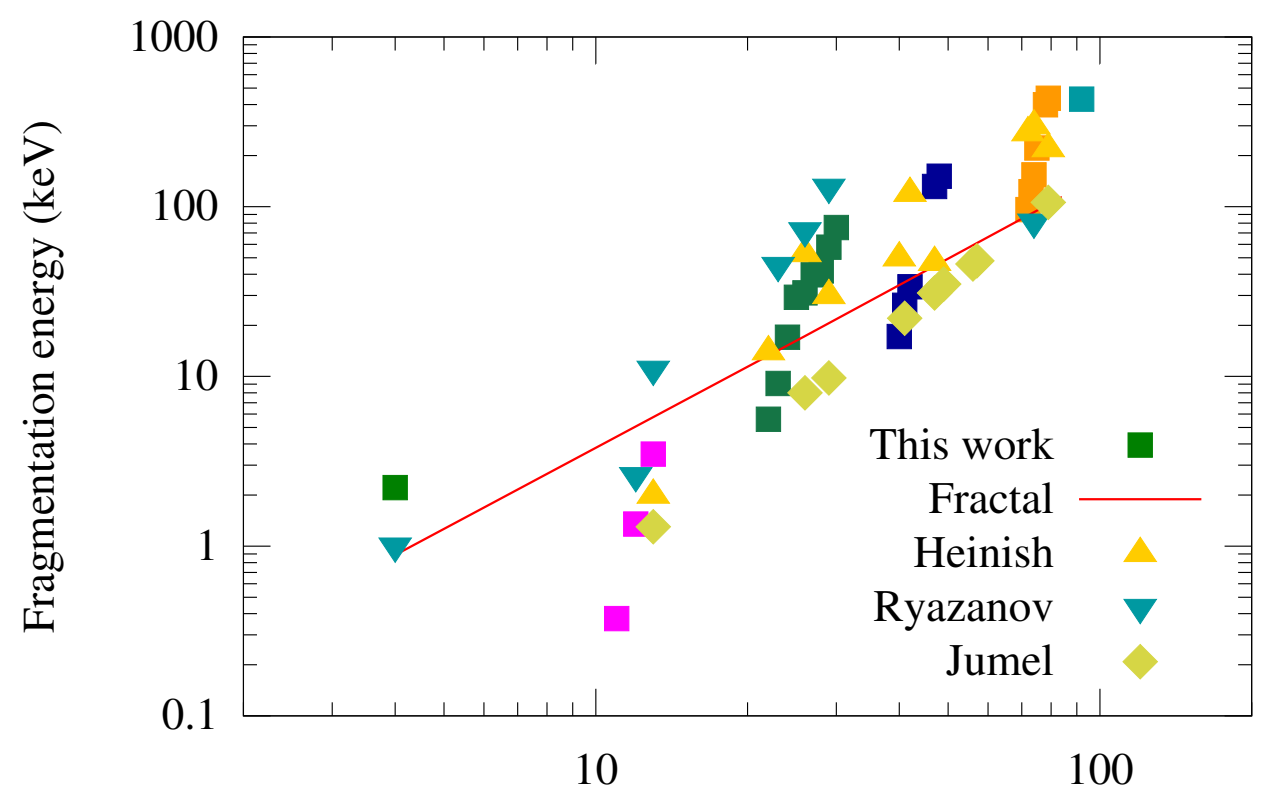

Atomic number, $\mathrm{Z}$

(b)

Figure 4: (a) Fragmentation energy as a function of the atomic number times the atomic density with $E_{c}=0$ and the adjusted power law given by equation (3). (b) Fragmentation energy, with $E_{c}$ given by the formula (2), compared to our fractal approach [36], the self consistent theory of Ryazanov [9], the break-up energy reported by Heinisch [7] and the analytic model of INCAS reported by Jumel [8]. Our results have been obtained with BCA cascades. 


\section{Subcascade spatial correlation}

In this section, we further study the cascade morphology and derive the number of pairs of subcascades likely to interact because they are at close distance from each other. In the next section we will propose the resulting correction term of our model of primary damage.

We used BCA cascades from $1 \mathrm{keV}$ to $1.4 \mathrm{MeV}$, with 1500 cascades per PKA energy in Fe. The centres and volumes of the subcascades form random multitype point patterns. Fe is a particularly interesting metal as the fragmentation energy is relatively low, and both sides of the transition are covered by the energy range studied here. Fe BCA cascades described as multitype point patterns of energy from $1 \mathrm{keV}$ to $1.4 \mathrm{MeV}$ are illustrated in Figure 5. For simplicity, subcascades are divided into 5 subgroups depending on their volume. The subgroups are named XS, S, M, L and XL and are respectively coloured in blue, cyan, green, yellow and red, with the size limit given in Table 2 . One can describe the growth of the random multitype point patterns as function of the energy as follows. At low energy they consist of one small sphere. When the E increases, the spheres first grow. Around $15 \mathrm{keV}$, small decorations start to appear in some patterns. At higher energy, multiple spheres of various size are visible at random distance from each other. Above $100 \mathrm{keV}$, small spheres tend to align along branches and large ones are found at the intersections or ends of branches.

The cross pair correlation function characterizes the spatial distribution of points composing random multi-type patterns. It is used in other studies of multicomponent systems, like the modelling of the structure of $\mathrm{GeO}_{2}$ glasses [38, 39], the analysis of atom probe tomography data [40], the study of different species of trees [41] or other examples of spatial point patterns [42, 43]. It quantifies the frequency of objects as a function of their mutual distance and we will use it to number the interacting subcascades considering a vicinity criterion.

Because of the continuous variation of the subcascade volume, the cross pair correlation function depends on 4 continuous variables, $g\left(r, v_{i}, v_{j}, E\right)$ where $r$ is the distance between the centres, $v_{i}$ and $v_{j}$ are the subcascade volumes and $\mathrm{E}$ is the PKA energy. The centre of the subcascade is defined as $\overrightarrow{r_{i}}=\frac{1}{n_{i}} \sum_{k=1}^{n_{i}} \overrightarrow{\rho_{k}}$ where $\overrightarrow{\rho_{k}}$ are the positions of the $n_{i}$ cubic cells forming the subcascade after our decomposition method. One has $v_{i}=n_{i} v_{E C}$ where $v_{E C}$ is the volume of the cubic cell equal to $1 \mathrm{~nm}^{3}$. 


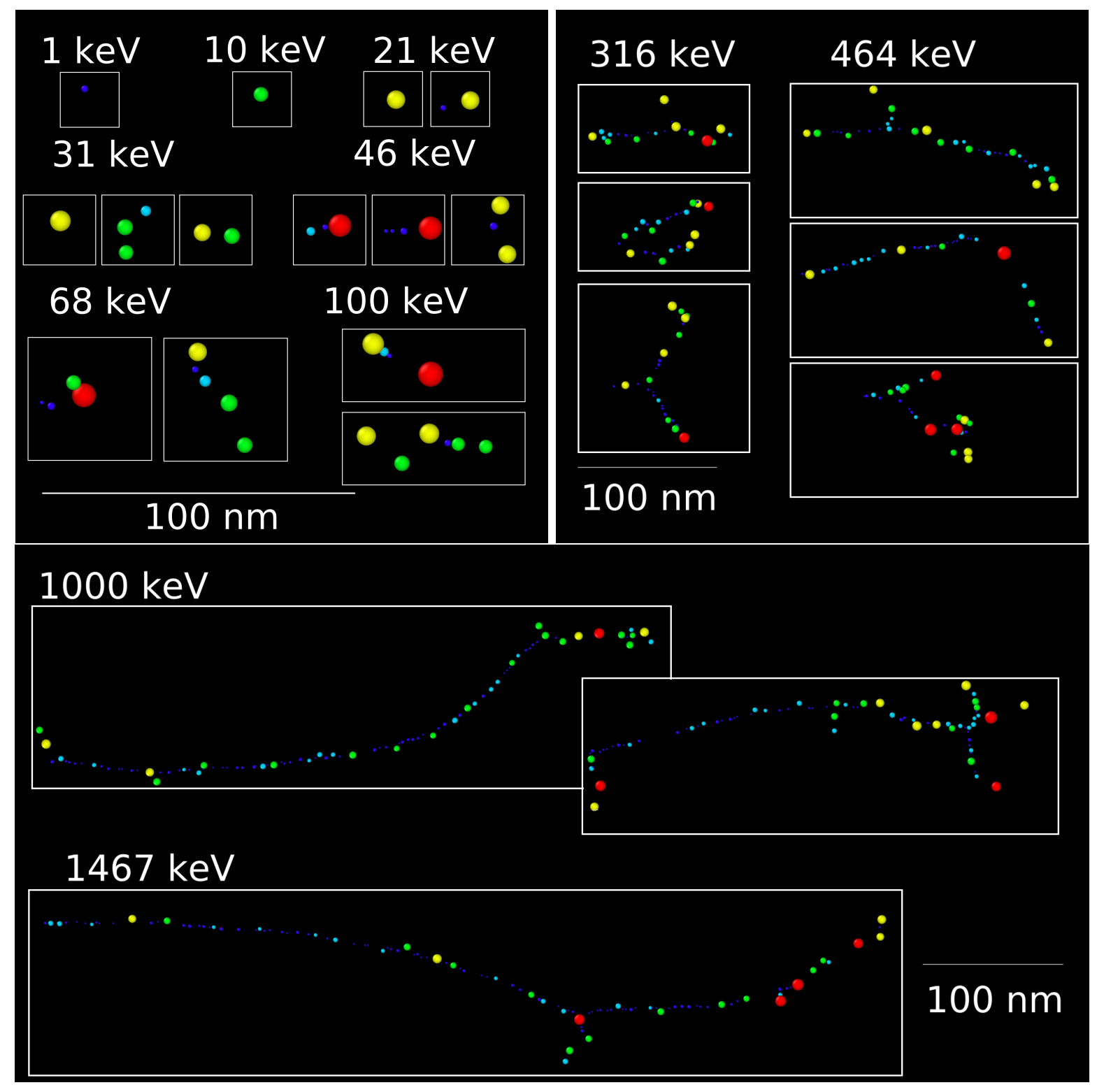

Figure 5: $1 \mathrm{keV}$ to $1.4 \mathrm{MeV}$ Fe BCA cascades analysed with our decomposition method and where subcascades are represented as spheres. The colours distinguish different subgroups based on a volume criterion given in Table 2. Blue, cyan, green, yellow and red are resp. XS, S, M, L and XL subcascades.

Figure 6 shows the total number of subcascades and the contributions of XS, S, M, L and XL subcascades as a function of the PKA energy. At low energy only one small sphere, successively of XS and S types, composes the cascade. At the transition where the number of subcascades increases, $\mathrm{M}$, then $\mathrm{L}$ subcascades appear, decorated by XS and S ones. Above the fragmentation energy, the number of subcascades increases linearly with the PKA energy. One sees the large contribution of XS and S subcascades in agreement with the power law distribution described in [15]. The total cascade volume and the different contributions are plotted in Figure 6b. As expected, only one type of subcascade mainly contributes to the cascade volume below transition. Above the transition, all categories contribute, however the L and XL subcascades contribute the most. 


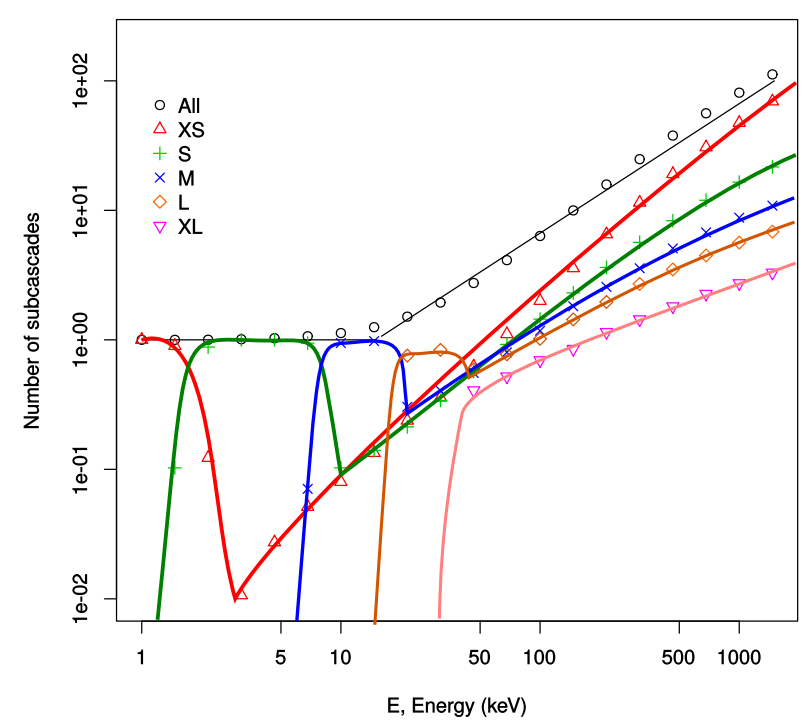

(a)

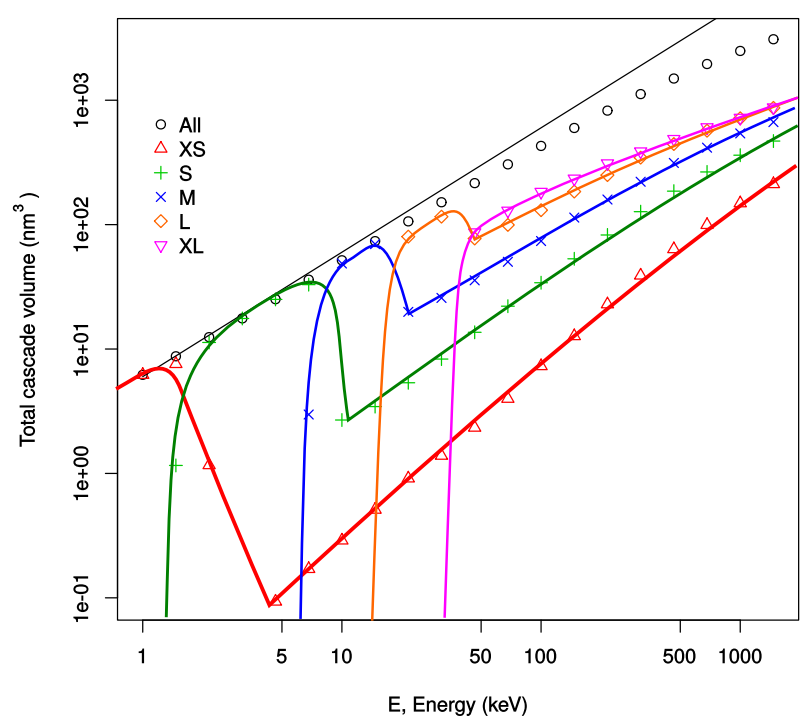

(b)

Figure 6: (a) Number of subcascades as function of the PKA energy in the XS, S, M, L and XL subgroups described in the table 2). The subcascade subgroups corresponds roughly to 1, 5, 10, 20 and $50 \mathrm{keV}$ cascades. (b) Total volume of cascades as function of the PKA energy and the contributions of the XS, S, M, L and XL subcascades. Lines are guides for the eyes. The number of subcascades is proportional to the energy over the fragmentation energy and the cascade volume is proportional to the energy, slightly sub-linear because of the electronic stopping power. BCA cascades have been used for this analysis.

Using these subcascade subgroups, cross pair correlation functions can be calculated as

$$
G_{i, j}(r, E)=\frac{1}{4 \pi r^{2} d r} \frac{1}{n_{c}} \sum_{k=1}^{n_{c}}\left(\frac{1}{2 n_{i, k} n_{j, k}} \sum_{i^{\prime}=1}^{n_{i, k}} \sum_{j^{\prime}=1}^{n_{j, k}} H\left(\left|\overrightarrow{r_{i^{\prime}}}-\overrightarrow{r_{j^{\prime}}}\right|-r\right) H\left(r+d r-\left|\overrightarrow{r_{i^{\prime}}}-\overrightarrow{r_{j^{\prime}}}\right|\right)\right)
$$

where $i$ and $j$ can be XS, S, M, L or XL, $r$ is the distance between subcascades, $d r$ is the bin width of $r$, $n_{i, k}$ is the number of points in subgroup $i$ in the cascade $k, H(x)$ is the Heaviside step function and $n_{c}$ is the number of cascades. 


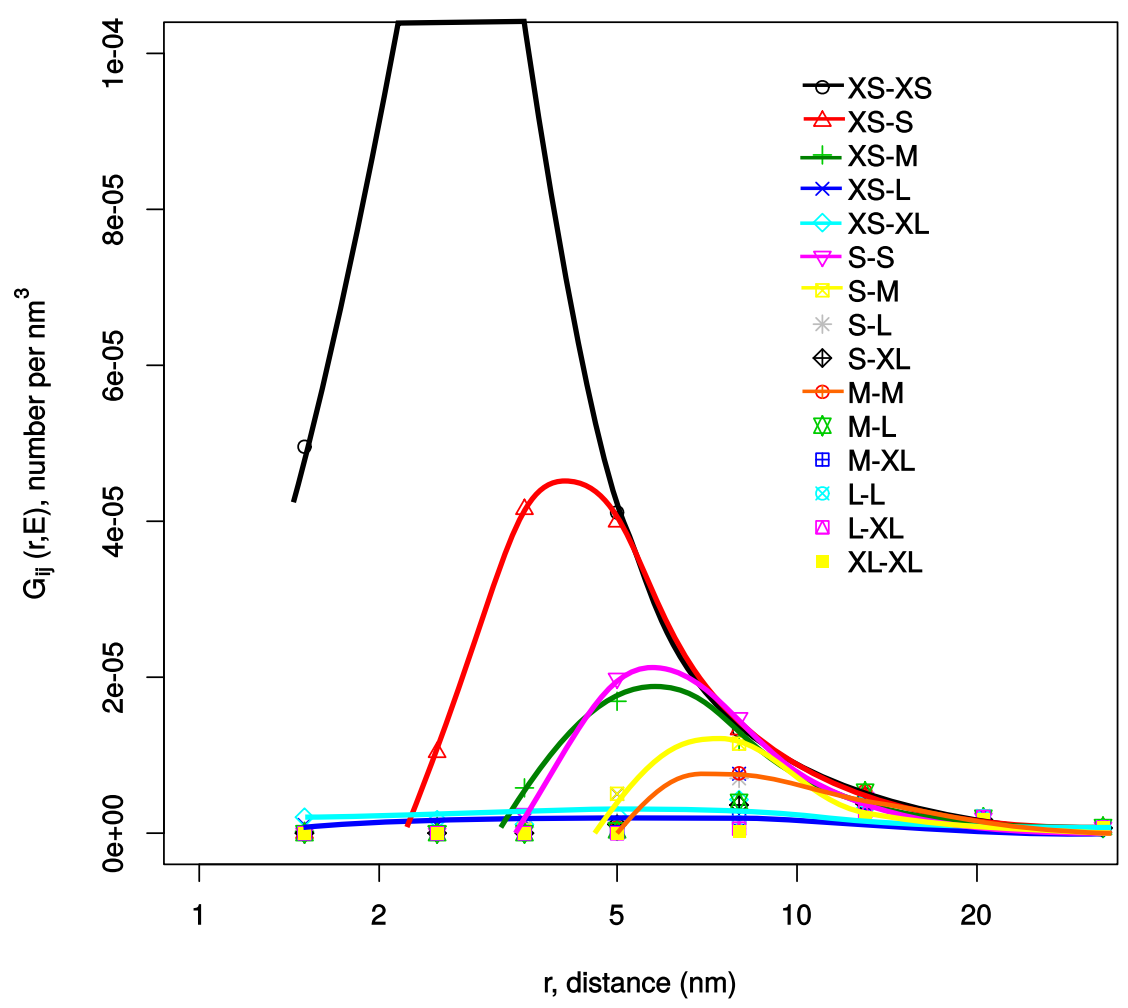

Figure 7: Cross pair correlation functions versus the distance between XS, S, M, L and XL subcascades for $E=500 \mathrm{keV}$ and BCA cascades.

We analysed the 15 cross pair correlation functions for each PKA energy. Figure 7 shows only the cross pair correlation functions of the $500 \mathrm{keV}$ cascades in Fe. They exhibit a maximum as function of $r$. The position of this maximum depends on the $\mathrm{i}, \mathrm{j}$ pair. The main reason is that our decomposition method excludes the possibility of overlap imposing a minimum distance between subcascades. On the other side, the whole cascade is enclosed in a limited volume, which imposes the pair correlation function to vanish when $r$ increases. Notice that $G_{X S, L}(r, E)$ is not negligible at short distance because the shape of a large subcascade is not spherical and there is a probability that one XS subcascade is almost enclosed in a large one. The position of the maximum roughly increases with the subcascade volume, for example $3 \mathrm{~nm}$ for XS-XS subcascades and $6 \mathrm{~nm}$ for the S-S subcascades.

When the number of subcascades per PKA becomes larger than 2, the subcascade interaction is possible. For $n_{S C}(E)$ subcascades, the number of pairs is simply $\frac{1}{2} n_{S C}(E)\left(n_{S C}(E)-1\right)$. As the number of subcascades is almost proportional to the energy (see equation (1)), the total number of interacting subcascades is

$$
N^{t o t}(E)=\frac{1}{2} \frac{E}{E_{f r}}\left(\frac{E}{E_{f r}}-1\right) \propto E^{2}
$$

which is approximately proportional to the square of the energy but does not detail the type of subcascades, and does not take into account their mutual distance. It is reasonable to consider that only subcascades close to each other will interact. The frequency of interacting subcascades is then reduced to integration of the cross pair correlation function on a limited distance interval. We propose a simple estimate of the number of interacting subcascades of volume $v_{i}$ and $v_{j}$ in cascades of energy $\mathrm{E}$, equal to

$$
N_{i, j}(r, E)=4 \pi r^{2} G_{i, j}(r, E) n_{i}(E) n_{j}(E) \Delta r
$$

where $n_{i}(E)$ is the averaged number of subcascades of volume $v_{i}$ in cascades of energy $\mathrm{E}$ and $\Delta r$ is the distance range where the interaction is significant. We naturally propose to take $r=r_{i, j}^{\max }$ where the cross pair correlation functions are close to the maximum and we observed that

$$
\begin{aligned}
r_{i, j}^{\max } & \sim R_{i}+R_{j}+\Delta r \\
\text { with } R_{i} & =\left(\frac{3}{4 \pi} v_{i}\right)^{\frac{1}{3}}
\end{aligned}
$$




\begin{tabular}{c|ccccc} 
i or $\mathrm{j}$ & $\mathrm{XS}$ & $\mathrm{S}$ & $\mathrm{M}$ & $\mathrm{L}$ & $\mathrm{XL}$ \\
\hline Volume $\left(\mathrm{nm}^{3}\right)$ & $0-10$ & $10-40$ & $40-90$ & $90-180$ & $180-1500$ \\
$R_{i}(\mathrm{~nm})$ & 1.1 & 1.7 & 2.5 & 3.5 & 5 \\
Energy $(\mathrm{keV})$ & 1 & 5 & 10 & 20 & 50 \\
\hline for E = 500 keV & & & & & \\
averaged $n_{i}^{k}$ & 19 & 8 & 5 & 3 & 2 \\
$N_{X S, j}$ & 0.51 & 1.0 & 0.09 & 0.11 & 0.025 \\
$N_{S, j}$ & & 0.027 & 0.067 & 0.013 & 0.044 \\
$N_{M, j}$ & & & 0.006 & 0.057 & 0.01 \\
$N_{L, j}$ & & & & 0.014 & 0.003 \\
$N_{X L, j}$ & & & & & 0.012
\end{tabular}

Table 2: Description of the subcascades distributed in 5 subgroups of different volume, XS, S, M, L, XL. Radius and PKA energy that can be associated with each group. For $500 \mathrm{keV}$ Fe cascades, averaged $n_{i}^{k}$ (number of subcascades of group i per cascade) and $N_{i, j}$, averaged numbers of interaction of subcascades for all cross pairs, XS-XS, XS-S, ..., XL-XL (see equation (8)).

where $R_{i}$ is our definition of the subcascade dimension and $\Delta r$ is $1 \mathrm{~nm}$ which is also the size of the cubic cells used in our decomposition method. The number of pairs of effectively interacting subcascades of size $i, j$ is finally

$$
N_{i, j}(E)=\left(\frac{9}{\sqrt{4 \pi}} v_{i} v_{j}\right)^{\frac{2}{3}} \Delta r G_{i, j}\left(r_{i, j}^{\max }, E\right) n_{i}(E) n_{j}(E) .
$$

This simplification will be also justified in the next section, where the effect of the subcascade interaction on the primary damage will be discussed

Using the equations (5) and (8), we can study the number of interacting subcascades as a function of the PKA energy and the $i, j$ pairs and some of them (XS-S, M-L and XL-XL) are plotted in Figure 8. With our estimate, the number of interacting subcascades is several times smaller than the total number, $N^{\text {tot }}(E)$. The difference increases with energy because the fragmentation of high energy cascades in a larger volume reduces the probability of pairs of subcascades at short distance from each other. Interestingly, the number of XS-S pairs which is an important term of the sum evolves proportionally to $E^{2}$ around the fragmentation energy and becomes $\propto E$ at high energy. This is a feature of the branching of the cascades. Indeed, the high energy cascades are made of a number of XS and S subcascades $\propto E$ which are found along the branches formed by the fast knock-on atoms (see blue small spheres in Figure 5). The number of close pairs is then proportional to $E$. The number of medium and large interacting subcascades is $\propto E^{s}$ with $s$ smaller than 1 and equal to 0.5 and 0.25 for M-L and XL-XL. This is due to the sub-linear increase of the number of these subcascades as function of the PKA energy and the reduction of the cross pair correlation function due to the expansion of the cascades in space. 


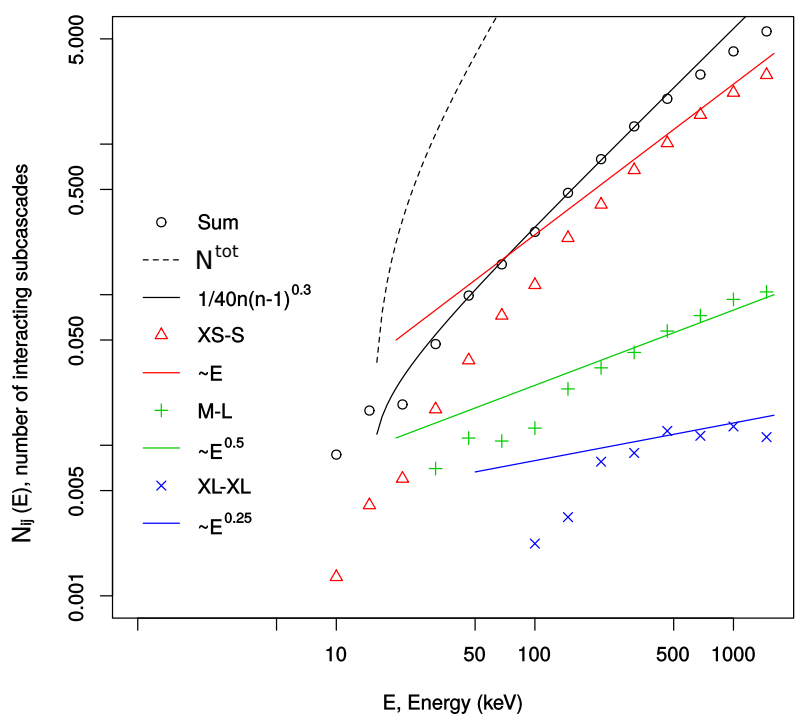

Figure 8: Number of XS-S, M-L and XL-XL pairs of interacting subcascades per cascade as a function of the PKA energy calculated with equation (8). Comparison with the maximum number of pairs, $N^{\text {tot }}$ (equation (5)). $\mathrm{BCA}$ cascades in $\mathrm{Fe}$ have been used for these results.

\section{Subcascade interaction and primary damage}

In the previous section, we studied and characterized the cascade morphological transition and evolution with the PKA energy using our subcascade decomposition method. We proposed a simple expression of the number of interacting pairs of subcascades of size i, j. We now include the subcascade interaction in our model of the primary damage.

We will first summarize the key features of the evolution of cascade morphology with energy, then describe our method to take them into account in a model of defect and defect cluster production for cascades of any energy. We will demonstrate the relevance of the subcascade interaction and adjust the model by direct comparison with defect production in high energy cascades in Fe calculated by full MD. Finally, we will predict the defect cluster size distribution at even higher energy when MD cascades are not available.

As a premise, Figure 9 illustrates two $80 \mathrm{keV}$ MD cascades. The subcascades at the maximum volume expansion (formed by cubes of different colours) are superposed to the large spheres of the multitype point pattern and to the defects remaining at the end of the cascade (small white and pink spheres). One sees the vacancy defects (in white) mainly in the centre of the subcascades. The small SIA defects (magenta) are found around the subcascades. Interestingly, a large SIA loop is found between one L and one XL subcascade. 


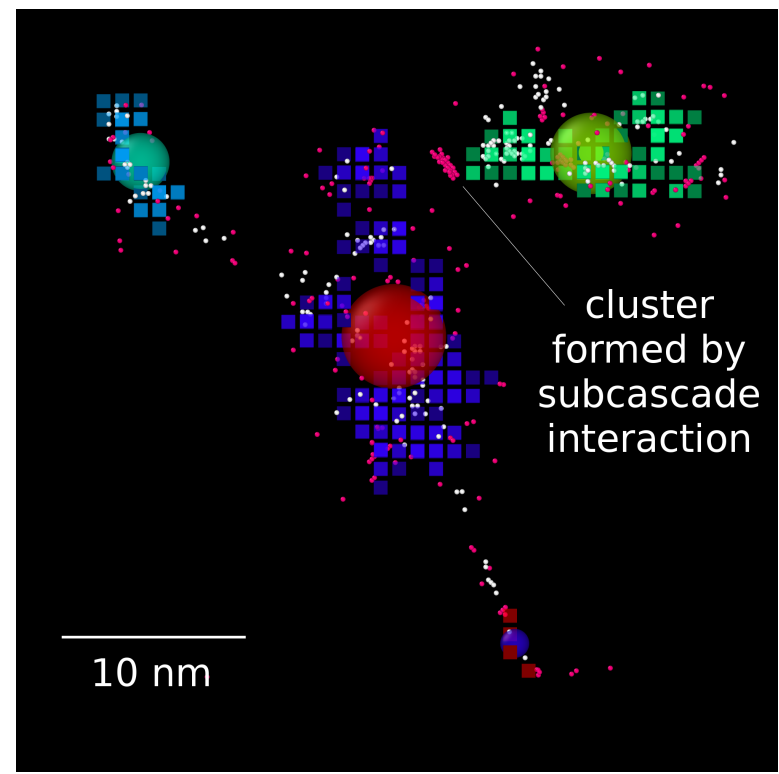

(a)

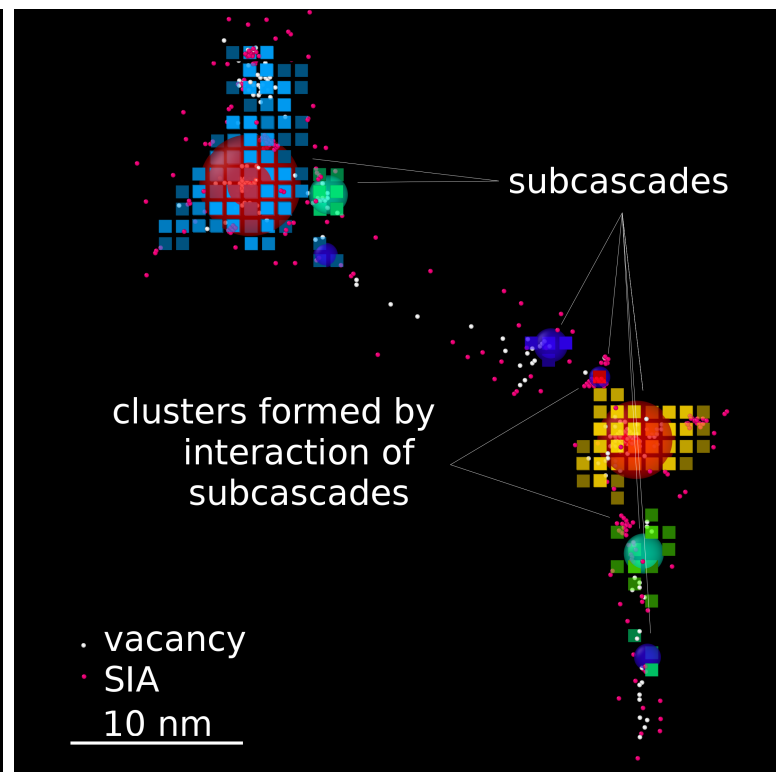

(b)

Figure 9: Two examples of $80 \mathrm{keV}$ MD cascades in Fe. Our subcascade decomposition method at the maximum expansion of the cascade determined 4 (in (a)) and 8 subcascades (in (b)) illustrated by cubes of different colours. The associated multitype point patterns are schematized by large spheres: blue, cyan, green, yellow and red are resp. XS, S, M, L and XL subcascades. Defects remaining after the cooling of the cascades are superposed as small white for vacancies and magenta spheres for SIAs. Vacancy defects are found mainly in the cores of the subcascades. The SIA defects are found at the periphery of the subcascades. Particularly large SIA clusters are indicated between two L and XL subcascades.

When the PKA energy increases, the probability of large energy secondary knock-on atoms that create subcascades that do not overlap, becomes possible. This causes the spatial fluctuations of the density of energy that we use to detect the cascade fragmentation in two or more intense cores of subcascades, separated by interfaces of no or low energy. The atoms tend to be ejected from the hot cores to their periphery. Interfaces are thus susceptible of collecting atoms ejected from two different cores. Moreover, shock-waves are emitted by the cascade core and are likely to interact at interfaces.

When the PKA energy increases, the average number of subcascades per cascade is first constant and equal to one, then proportional to the energy above the fragmentation energy, $E_{f r}$. The statistics of the subcascades as a function of their volume reveals another aspect of the morphological transition described in [15]. At low energy, the distribution can be described by a peak around the averaged volume and a power law of small volumes that represents rare small subcascades decorating the main one. The position of the peak moves toward large volumes when the PKA energy increases. The power law contribution also increases with the PKA energy. Above the transition, the peak disappears and only the power law is visible and continues to increase. A deviation to the power law is observed when the frequency of subcascades vanishes, which determines a maximum subcascade volume, as described in $[15,16]$. The maximum subcascade volume slowly increases with the energy up to 2-3 times the fragmentation volume. The total volume of the cascade remains proportional to the PKA energy. The number of subcascades as described in the previous section can be obtained by integration of the distribution. One has

$$
\begin{array}{r}
V(E)=\int v D(v, E) d v=V_{f r} \frac{E}{E_{f r}} \\
n_{S C}(E)=\int D(v, E) d v
\end{array}
$$

where $V(E)$ is the total volume, $D(v, E)$ is the distribution of subcascades and $V_{f r}$ and $E_{f r}$ are the fragmentation volume and energy.

In [13], we proposed that the total defect production at any PKA energy, $P(E)$, is the sum of the defect production in individual subcascades. In [15], we extended this approach to the different contributions of clusters of n defects $p(n, E)$. One has

$$
P(E)=\int p(n, E) n d n
$$

At this stage, we do not know if the interaction of subcascades will increase or decrease the number of defects of size $\mathrm{n}$. We introduce the subcascade interaction as pairwise terms and the production of defects of size $n$ 
becomes

$$
\begin{aligned}
p(n, E) & =f_{1}(n, E)+f_{2}(n, E) \\
& =\int f_{1}(n, v) D(v, E) d v \\
& +\iiint f_{2}\left(n, v_{i}, v_{j}, r\right) S\left(E, v_{i}, v_{j}, r\right) d v_{i} d v_{j} d r
\end{aligned}
$$

where $f_{1}(n, E)$ is the defect production in individual subcascades and $f_{2}(n, E)$, the subcascade interaction pairwise terms. $f_{2}\left(n, v_{i}, v_{j}, r\right)$ is the interaction of two subcascades of volume $v_{i}$ and $v_{j}$ at distance $r$ from each other and $S\left(E, v_{i}, v_{j}, r\right)$ the averaged number of pairs of subcascade of volume $v_{i}$ and $v_{j}$ at a distance $r$ in cascades of energy E. Subcascades are described in discrete subgroups of volumes, XS, S, M, L and XL and in the previous section, we detailed the expression (6) which means that

$$
S\left(E, v_{i}, v_{j}, r\right) \sim N_{i, j}(r, E)
$$

where $N_{i, j}(r, E)$ was simplified in $N_{i, j}(E)$ (see equation (8)). Here also, $f_{2}\left(n, v_{i}, v_{j}, r\right)$ depends on the distance between subcascades and should also vanish at large distance. This suggests a similar simplification and equation (11) simplifies in

$$
\begin{aligned}
N_{i, j}(E) p(n, E) & \sim \int f_{1}(n, v) D(v, E) d v+\sum_{i, j} f_{2, i, j}(n) N_{i, j}(E) \\
\text { with } f_{2, i, j}(n) & \sim f_{2}\left(n, v_{i}, v_{j}, r_{i, j}^{\max }\right)
\end{aligned}
$$

where the second term is a sum of the product of the elements of two matrices, the first one gives the interaction of two subcascades of volume $i$ and $j$ at a distance $r_{i, j}^{\max }$, and an example of the second one is given in Table 2 for $E=500 \mathrm{keV}$.

Different approaches are surely possible to determine $f_{2, i, j}(n)$ as for example, the overlap study of [44]. For our work, after analysis of the defect production by MD cascades we propose functional forms for $f_{1}(n, v)$ and $f_{2, i, j}(n)$ with a limited number of parameters. Like in $[15,16]$, one has

$$
\begin{gathered}
f_{1}(n, v)=\frac{A(v)}{n^{S}}\left(1-\frac{n}{n^{\max }(v)}\right) \\
\text { with } A(v)=A_{f r}\left(\frac{v}{v_{f r}}\right)^{T} \\
\text { and } n^{\max }(v)=n_{f r}^{\max }\left(\frac{v}{v_{f r}}\right)^{U}
\end{gathered}
$$

where $\mathrm{S}$ is the exponent of the power law already observed in [14], $n^{\max }(v)$ is the maximum defect size that can be created in a subcascade of volume $v$ and $n_{f r}^{\max }$ is the maximum cluster size at the fragmentation energy. A convenient functional form for $f_{2, i, j}(n)$ is

$$
\begin{aligned}
f_{2, i, j}(n) & =\frac{F_{i, j}}{n^{\alpha}}\left(\delta_{1, n}+\cos \left(\frac{3 \pi}{2}\left(\frac{\log (n)}{\log \left(n_{i, j}^{\text {max }}\right)}\right)^{\beta}\right)\right) \\
F_{i, j} & =\gamma\left(\frac{\sqrt{v_{i} v_{j}}}{V_{f r}}\right)^{\epsilon} \\
n_{i, j}^{\max } & =\zeta\left(\frac{\sqrt{v_{i} v_{j}}}{V_{f r}}\right)^{\kappa}
\end{aligned}
$$

where $n_{i, j}^{\max }$ is the maximum defect size impacted by the interaction of two subcascades of volume $v_{i}$ and $v_{j}$. The cosine (with the logarithmic argument) leads to a positive and a negative part with a zero at an adjustable position. $\delta_{1, n}$ is the Kronecker delta and the power law of $n$ is similar to $f_{1}(n, v)$. In equation (15), we assume that $F_{i, j}$ increases with the subcascade volume because a larger effect is expected from the interaction of a pair of large subcascades than small subcascades. Notice that this functional form is adimensional, symmetric in $i$ and $j$ and determined by 6 parameters, $\alpha, \beta, \gamma, \epsilon, \kappa$ and $\zeta$. Also, $f_{2, i, j}(n)$ is independent of $E$ and can be adjusted on the defect production of PKA energy larger than the fragmentation energy.

For this work, we applied our approach using partially the data base of MD cascades in Fe from [25]. Further results will be reported separately. Below the fragmentation energy, the defect cluster production follows a simple power law with the expected deviation due to the maximum defect size. At high energy, a hump is visible for the large defects and we will see that it cannot be explained by the production of individual subcascades. We adjusted the parameters of $f_{1}(n, v)$ given by equation (14) on these low energy cascades (from 1 to $10 \mathrm{keV}$ ) using Openturns [45]. The 6 parameters of $f_{2, i, j}(n)$ has been adjusted using the $80 \mathrm{keV}$ 


\begin{tabular}{cc|cc}
$f_{1}(n, E)$ & & $f_{2}(n, E)$ & \\
$S$ & 2.77 & $\alpha$ & 2.2 \\
$A_{f r}$ & 22 & $\beta$ & 1.7 \\
$T$ & 0.61 & $\gamma$ & 950 \\
$n_{f r}^{\max }$ & 61 & $\epsilon$ & 0.8 \\
$U$ & 0.82 & $\zeta$ & 75 \\
& & $\kappa$ & 0.9
\end{tabular}

Table 3: Parameters of the model of primary damage, $p(n, E)$, that includes two terms: $f_{1}(n, E)$, the defect production of subcascades taken individually and $f_{2}(n, E)$, the correction due to the subcascade interaction. Notice that the fragmentation energy and volume have been determined in the first section, $E_{f r}=15 \mathrm{keV}$ and $V_{f r}=90 \mathrm{~nm}^{3}$.

MD cascades. Because the electronic stopping power is included in our BCA cascades and not in the MD cascades, we use the matrix $N_{i, j}(E)$ of the $100 \mathrm{keV}$ BCA cascades. The parameters are given in Table 3.

We are now able to extrapolate our model of primary damage to $500 \mathrm{keV}$ cascades, using the morphological description given by the BCA. Both contributions, i.e. the defect production of subcascades taken individually, and the correction due to the subcascade interaction, have been calculated. In Figure 10a, we plotted the defect production calculated with and without subcascade interaction for the $5 \mathrm{keV}, 80 \mathrm{keV}$ and $500 \mathrm{keV}$, and compared to the defect production from the MD cascades. The defect production in $5 \mathrm{keV}$ cascades where the subcascade interaction is insignificant is correctly given by the main term $f_{1}(n, E)$. For the 80 $\mathrm{keV}$ cascades, the subcascade interaction causes a reduction of the frequency of the small defects and a hump of the large defects. In Figure $10 \mathrm{~b}$ the functions $f_{2, i, i}(n)$ are plotted. One sees that they are first negative then positive when $n$ increases, which causes the reduction of the production of small clusters, and the increase of the production of large clusters when two subcascades interact. The positions of the zero and the maximum cluster size increase with the volume of the interacting subcascades. The consequence is visible on the extrapolation to the primary damage of the $500 \mathrm{keV}$ cascades, where our model predicts an increase of the fraction of clusters due to the subcascade interaction.

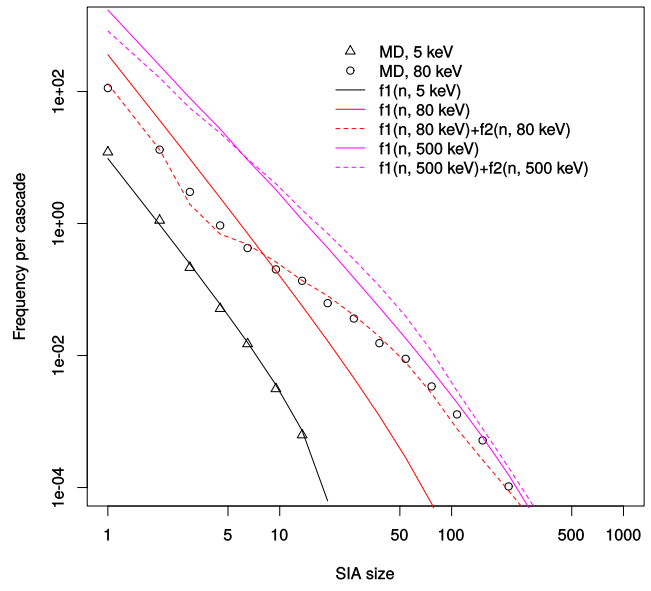

(a)

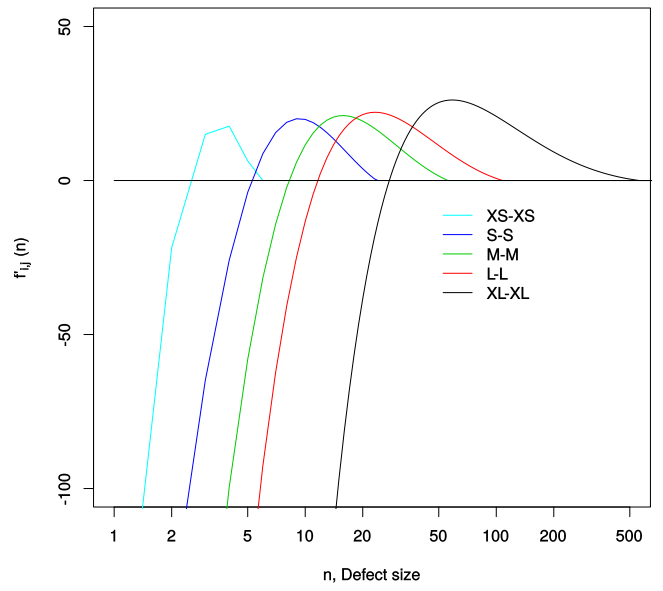

(b)

Figure 10: (a) The comparison of defect cluster frequencies from MD and our model with and without the subcascade interaction for $5 \mathrm{keV}$ and $80 \mathrm{keV}$ cascades and our model prediction for $500 \mathrm{keV}$ cascades based on the morphology given by the BCA only. (b) $f_{2, i, j}(n)$ functions of the subcascade interaction used in (13).

\section{Discussion}

We proposed a method that decomposes any cascade in subcascades with no overlap. We described how two parameters, the cell size and the energy criterion influence the subcascade decomposition. The method has been applied to BCA and MD cascades and it appears that the number of subcascades is quite independent of the cell size in a wide range of value from $1 \mathrm{~nm}$ to $2 \mathrm{~nm}$. With a strict criteria considering no collision 
in the subcascade interface $\left(E_{c}=0\right)$, we found that the fragmentation energy is a power law of an effective atomic number given by the product of the atomic number and the atomic density, due to the fractal nature of the cascades. To improve the subcascade interface detection, we proposed a less strict criterion, $E_{c}$ given by a simple formula (2) to estimate $E_{c}$ and applied our method to more than twenty metals. It decreases the fragmentation energy to values which are in agreement with MD and experimental results on $\mathrm{W}$. The fragmentation energy of $\mathrm{W}$ is $75 \mathrm{keV}$ with $E_{c}=76 \mathrm{eV} / \mathrm{nm}^{3}$. Still these values slightly vary with the cell size and the energy criterion. There is no well-established definition of the beginning of cascade fragmentation. Our method shown that this is a continuous process with the increase of the frequency of small decorations around the main subcascade. Clear features of the subcascade decomposition above the fragmentation energy are the disappearance of any specific subcascade volume and the existence of a maximum subcascade volume. Indeed, the distribution of subcascade volume turns from a peak to a power law that vanishes for the maximum subcascade volume (described in $[15,16]$ ). Comparison between MD and BCA cascades show a good agreement because the MD empirical potential has been adjusted on the BCA ZBL potential on the short distance range, that intervenes during the ballistic stage of the cascades. Notice that atoms in MD cascades can experience channelling due to the crystal structure, and this is not accounted for in our BCA model. The channelling effect has been studied with other BCA codes that account for crystal structure, MARLOWE in [46] and MDRANGE compared to MD in [47]. Another difference is that in high energy MD cascades, small subcascades can disappear before the end of the ballistic stage. It must be added that we observed a visible effect of the empirical potential of MD cascades on the number of subcascades. These potentials have different equilibrium properties or have been bridged to the ZBL potential differently [16]. Further work is necessary to explain the exact causes of these variations and determine the relation with the energy criterion $E_{c}$, which could include other thermodynamic properties like the thermal diffusivity. The BCA is still a convenient method to reach the number of trials necessary for good statistics, especially at high energy.

Subcascades can be seen as forming a multitype point pattern characterised by cross pair correlation functions. With no loss of generality we proposes to simplify the volume, which in principle evolves in continuous way, in discrete categories, reducing integrals into sums. The cross pair correlation functions exhibit a maximum because of the minimum distance required by the no overlap criterion and because the cascade is enclosed in a finite volume. Still the number of interacting pairs of subcascades is in principle given by the integral of the cross pair correlation function on a range of distance (and subcascade volume) but we show that only close pairs are of interest. We propose a strong simplification taking the value of the cross pair correlation functions at their maximum multiplied by a thickness of $1 \mathrm{~nm}$. We then studied the number of interacting pairs of subgroups $i$ and $j$ in cascade of many PKA energies and their evolution with the PKA energy. The branching of cascades where their morphology is formed by elongated tracks decorated by small subcascades, which causes that the number of interactions of small subcascades increases proportionally to the PKA energy as their form chaplets decorating the branches, whereas the number of medium (resp. large) subcascades pairs increases as square root of $\mathrm{E}$ (resp. $\left.E^{1 / 4}\right)$.

We included the number of interacting subcascades in our model of primary damage. In [15], a first term was determined for the defect cluster production in individual subcascades. Here pairwise terms have been added that correspond to the effect of subcascade interactions on the defect production. Three body terms could in principle be included and other mechanism of enhanced cluster formation cannot be excluded. Indeed, in [16], for example, we observed a change of the power law slope of defect cluster production in W cascades of energy smaller than the fragmentation energy. We proposed functional forms for the unknown features and ended up with a model with a limited number of parameters and physical meaning. Using our large database or MD cascades from [25], we directly compared and adjusted our model to the defects created in MD cascades. We showed that below the fragmentation energy, the defect production is correctly given by the first term of our model. The defect production of high energy cascade is not correctly predicted by the defect production in individual subcascades only. We explained and illustrated that this is due to the interaction of subcascades which increases the cluster fraction, i.e. the reduction of the frequency of small clusters and the enhanced formation of large ones. We observed that the subcascade interaction stabilizes large SIA clusters, resulting in fewer small ones, than with isolated subcascades without creating significantly more defects. We adjusted interaction functions, which permits the prediction of the primary damage of the cascade whose energy is not accessible by full MD. More accurate results can be obtained by adjusting our model with more high energy cascades, or combining with other approaches to model the mechanism of these interactions. Further works are necessary to characterize the effect of subcascade interaction on the vacancy clusters. Other mechanisms could also impact the defect cluster production as the electronic losses and electron-phonon interaction.

One missing aspect of this work is the cascade description along its main direction. Indeed, in high energy ion irradiation, it is established that, the averaged damage distribution and the defect versus size frequency 
depends on the distance from the source (or surface) in the direction of the impinging particle. Finally, though our model takes partially into account the electronic stopping power, it does not account for this specific heat transport and its effect on the defect recovery.

\section{Conclusion}

Using MD and BCA calculations we studied the morphological transition of collision cascades in a large range of PKA energy. A transition from compact to fragmented cascades is observed in all metals and the mean features depend on the atomic number and the atomic density. The fragmentation energy follows a simple power law of these two parameters. We introduced an energy criterion that improves the sensitivity of our method especially in high $\mathrm{Z}$ materials, and includes thermodynamic properties such as the melting point temperature.

The distribution of subcascade volumes and the spatial correlation of the subcascades can be accurately and efficiently studied using the BCA and statistics of random multitype point patterns. We derived the number of interacting subcascades as function of their volume described as a limited number of subgroups. We have shown that when the cascade branching starts the number of interacting small-small subcascades increases proportionally to the PKA energy while the number of medium (resp. large) subcascades pairs increases as square root of $\mathrm{E}$ (resp. $\left.\mathrm{E}^{1 / 4}\right)$.

We developed our model of primary damage now including two terms: the production of defects in subcascades taken individually, and pairwise terms of the subcascade interaction. The model has been applied to Fe. We have shown that the subcascade interaction increases the SIA cluster fraction in high energy cascade. It has been used to extrapolate the defect production in even larger PKA energy, but it could also be convoluted with the neutron PKA spectrum. Our approach is also valid for other metals or materials.

\section{Acknowledgements}

This work was part-funded by the EPSRC Program [grant number: EP/P012450/1]. This work has been carried out within the framework of the EUROfusion Consortium and has received funding from the Euratom research and training program 2014-2018 under grant agreement No 633053. The views and opinions expressed herein do not necessarily reflect those of the European Commission. This work is supported by CEA/DEN under RSTB/MATIX research program. This work is part of the European project SOTERIA (661913). C. Domain and C.S. Becquart acknowledge the EM2VM, Joint laboratory Study and Modelling of the Microstructure for Ageing of Materials. The contribution of A. De Backer is in the frame of the Enabling Research project TriCEM on Tritium Retention in Controlled and Evolving Microstructure.

\section{References}

[1] K. H. Nordlund, A. E. Sand, F. G. Granberg, S. Zinkle, R. Stoller, R. Averback, T.Suzudo, L. Malerba, F. Banhart, W. Weber, F. Willaime, S. Dudarev, and D. Simeone. Primary Radiation Damage in Materials: Review of Current Understanding and Proposed New Standard Displacement Damage Model to Incorporate in Cascade Defect Production Efficiency and Mixing Effects. OECD Nuclear Energy Agency, 2015.

[2] C. Björkas, K. Nordlund, and M. J. Caturla. Influence of the picosecond defect distribution on damage accumulation in irradiated $\alpha$-fe. Phys. Rev. B, 85:024105, Jan 2012.

[3] G. Adjanor, S. Bugat, C. Domain, and A. Barbu. Overview of the RPV-2 and intern-1 packages: From primary damage to microplasticity. Journal of Nuclear Materials, 406(1):175 - 186, 2010.

[4] M.R. Gilbert, J.-Ch. Sublet, and S.L. Dudarev. Spatial heterogeneity of tungsten transmutation in a fusion device. Nuclear Fusion, 57(4):044002, 2017.

[5] M. Hou. Fuzzy clustering methods: An application to atomic displacement cascades in solids. Phys. Rev. A, 39:2817-2828, Mar 1989.

[6] Y. Satoh, S. Kojima, T. Yoshiie, and M. Kiritani. Criterion of subcascade formation in metals from atomic collision calculation. Journal of Nuclear Materials, 179:901 - 904, 1991.

[7] H. L. Heinisch and B. N. Singh. On the structure of irradiation-induced collision cascades in metals as a function of recoil energy and crystal structure. Philosophical Magazine A, 67(2):407-424, 1993. 
[8] S. Jumel and J. C. Van-Duysen. Incas: an analytical model to describe displacement cascades. Journal of Nuclear Materials, 328(23):151 - 164, 2004.

[9] A.I. Ryazanov, E.V. Metelkin, and E.V. Semenov. Modeling of cascade and sub-cascade formation at high $\{$ PKA $\}$ energies in irradiated fusion structural materials. Journal of Nuclear Materials, 386 - 388:132 - 134, 2009. Fusion Reactor MaterialsProceedings of the Thirteenth International Conference on Fusion Reactor Materials.

[10] L. Lunéville, D. Simeone, and W.J. Weber. Study of the fragmentation of a displacement cascade into subcascades within the binary collision approximation framework. Journal of Nuclear Materials, 415(1):55 - 60, 2011.

[11] A.F. Calder, D.J. Bacon, A.V. Barashev, and Yu.N. Osetsky. On the origin of large interstitial clusters in displacement cascades. Philosophical Magazine, 90(7-8):863-884, 2010.

[12] W. Setyawan, A. P. Selby, N. Juslin, R.S. Stoller, B.D. Wirth, and R.J. Kurtz. Cascade morphology transition in bcc metals. Journal of Physics: Condensed Matter, 27(22):225402, 2015.

[13] L. Lunéville, D. Simeone, and C. Jouanne. Calculation of radiation damage induced by neutrons in compound materials. Journal of Nuclear Materials, 353(12):89 - 100, 2006.

[14] A. E. Sand, S. L. Dudarev, and K. Nordlund. High energy collision cascades in tungsten: dislocation loops structure and clustering scaling laws. European Physic Letters, 103:46003, 2013.

[15] A. De Backer, A. E. Sand, K. Nordlund, L. Lunéville, D. Simeone, and S. L. Dudarev. Subcascade formation and defect cluster size scaling in high-energy collision events in metals. EPL (Europhysics Letters), 115(2):26001, 2016.

[16] A. E. Sand, D. R. Mason, A. De Backer, X. Yi, S. L. Dudarev, and K. Nordlund. Cascade fragmentation: deviation from power law in primary radiation damage. Materials Research Letters, 5(5):357-363, 2017.

[17] X. Yi, A. E. Sand, D. R. Mason, M. A. Kirk, S. G. Roberts, K. Nordlund, and S. L. Dudarev. Direct observation of size scaling and elastic interaction between nano-scale defects in collision cascades. EPL (Europhysics Letters), 110(3):36001, 2015.

[18] W. Eckstein A. Mutzke, R. Schneider and R. Dohmen. SDTrimSP version 5.00, IPP Report12/08. MaxPlanck-Institut fr Plasmaphysik, 2011.

[19] W. Eckstein. Computer Simulation of Ion-Solid Interactions. 10. Springer Berlin Heidelberg, 1991.

[20] J. F. Ziegler, J. P. Biersack, and U. Littmark. The Stopping and Range of Ions in Matter. Pergamon, New York, 1985.

[21] J. F. Ziegler, M.D. Ziegler, and J.P. Biersack. \{SRIM $\}$ - the stopping and range of ions in matter (2010). Nuclear Instruments and Methods in Physics Research Section B: Beam Interactions with Materials and Atoms, 268(11 - 12):1818 - 1823, 2010.

[22] O. S. Oen and M. T. Robinson. Computer studies of the reflection of light ions from solids. Nuclear Instruments and Methods, 132:647 - 653, 1976.

[23] E. Zarkadoula, S. L. Daraszewicz, D. M. Duffy, M. A. Seaton, I. T. Todorov, K. Nordlund, M. T. Dove, and K. Trachenko. The nature of high-energy radiation damage in iron. Journal of Physics: Condensed Matter, 25(12):125402, 2013.

[24] E Zarkadoula, D M Duffy, K Nordlund, M A Seaton, I T Todorov, W J Weber, and K Trachenko. Electronic effects in high-energy radiation damage in tungsten. Journal of Physics: Condensed Matter, 27(13):135401, 2015.

[25] C. Domain, A. De Backer, and C. S. Becquart. in preparation.

[26] C. S. Becquart, K. M. Decker, C. Domain, J. Ruste, Y. Souffez, J. C. Turbatte, and J. C. Van Duysen. Massively parallel molecular dynamics simulations with eam potentials. Radiation Effects and Defects in Solids, 142(1-4):9-21, 1997.

[27] G J Ackland, M I Mendelev, D J Srolovitz, S Han, and A V Barashev. Development of an interatomic potential for phosphorus impurities in $\alpha$-iron. Journal of Physics: Condensed Matter, 16(27):S2629, 2004.

[28] P. Olsson, C. S. Becquart, and C. Domain. Ab initio threshold displacement energies in iron. Materials Research Letters, 4(4):219-225, 2016.

[29] R.E. Stoller, G.R. Odette, and B.D. Wirth. Primary damage formation in bcc iron. Journal of Nuclear Materials, 251:49, 1997. Proceedings of the International Workshop on Defect Production, Accumulation and Materials Performance in an Irradiation Environment. 
[30] A. V. Granato. Interstitial Model for Condensed Matter States of Face-Centered-Cubic Metals. Phys. Rev. Lett., 68(7):974, 1992.

[31] K. Nordlund and R. S. Averback. The role of self-interstitial atoms on the high temperature properties of metals. Phys. Rev. Lett., 80(19):4201-4204, 1998.

[32] K. Nordlund, Y. Ashkenazy, R. S. Averback, and A. V. Granato. Strings and interstitials in liquids, glasses and crystals. Europhys. Lett., 71(4):625, 2005.

[33] M. Forsblom and G. Grimvall. Homogeneous melting of superheated crystals: Molecular dynamics simulations. Phys. Rev. B, 72:054107, Aug 2005.

[34] G. K. White and S. J. Collocott. Heat capacity of reference materials: Cu and W. Journal of Physical and Chemical Reference Data, 13(4):1251-1257, 1984.

[35] P. Olsson, T. P. C. Klaver, and C. Domain. Ab initio study of solute transition-metal interactions with point defects in bcc fe. Phys. Rev. B, 81:054102, Feb 2010.

[36] D. Simeone, L. Lunéville, and Y. Serruys. Cascade fragmentation under ion beam irradiation: A fractal approach. Phys. Rev. E, 82:011122, 2010.

[37] M. T. Robinson and I. M. Torrens. Computer simulation of atomic-displacement cascades in solids in the binary-collision approximation. Phys. Rev. B, 9:5008-5024, Jun 1974.

[38] M. Micoulaut. Structure of densified amorphous germanium dioxide. Journal of Physics: Condensed Matter, 16(10):L131, 2004.

[39] D. Marrocchelli, M. Salanne, and P. A Madden. High-pressure behaviour of geo 2 : a simulation study. Journal of Physics: Condensed Matter, 22(15):152102, 2010.

[40] T. Philippe, S. Duguay, G. Grancher, and D. Blavette. Point process statistics in atom probe tomography. Ultramicroscopy, 132(Supplement C):114 - 120, 2013. IFES 2012.

[41] T. Wiegand and K. A. Moloney. Handbook of Spatial Point-Pattern Analysis in Ecology. Chapman \& Hall/CRC, 2013.

[42] J. Illian, A. Penttinen, H. Stoyan, and D. Stoyan. Statistical Analysis and Modelling of Spatial Point Patterns. Wiley, 2008.

[43] A. E. Gelfand, P. J. Diggle, M. Fuentes, and P. Guttorp. Handbook of spatial statistics. Chapman \& Hall/CRC, Boca Raton, 2010.

[44] D.J. Bacon, A.F. Calder, and F. Gao. Defect production due to displacement cascades in metals as revealed by computer simulation. Journal of Nuclear Materials, 251:1 - 12, 1997. Proceedings of the International Workshop on Defect Production, Accumulation and Materials Performance in an Irradiation Environment.

[45] Michaël Baudin, Anne Dutfoy, Bertrand Iooss, and Anne-Laure Popelin. Openturns: An industrial software for uncertainty quantification in simulation. 2015.

[46] M. Hou, C.J. Ortiz, C.S. Becquart, C. Domain, U. Sarkar, and A. De backer. Microstructure evolution of irradiated tungsten: Crystal effects in he and $\mathrm{h}$ implantation as modelled in the binary collision approximation. Journal of Nuclear Materials, 403(1 - 3):89 - 100, 2010.

[47] K. Nordlund, F. Djurabekova, and G. Hobler. Large fraction of crystal directions leads to ion channeling. Phys. Rev. B, 94:214109, Dec 2016. 